\title{
Homenaje a Stanley John Dudrick, MD, FACS (1935 - 2020)
}

\author{
A tribute to Stanley John Dudrick, MD, FACS \\ (1935 - 2020) \\ Homenagem a Stanley John Dudrick, MD, FACS
}

(1935 - 2020)

Vanessa Fuchs-Tarlovsky ${ }^{*}$

Recibido: 26 de enero 2020. Aceptado para publicación: 28 de enero 2020. https://doi.org/10.35454/rncm.v3n1.084

\author{
"Quiero dejar algo mejor cuando me vaya, en lugar de simplemente practicar \\ la medicina como siempre se ha hecho". \\ "I want to leave something better behind when I go, rather than just practice medicine \\ the way it has always been done." \\ "Quero deixar algo melhor para trás quando eu for, em vez de apenas praticar \\ medicina da maneira que sempre foi feita."
}

\section{S.J. Dudrick}

\begin{abstract}
Resumen
El Profesor Stanley J. Dudrick, considerado el padre de la nutrición parenteral o endovenosa fue pionero en utilizar esta vía para la administración de nutrientes. Sus primeros estudios en perros sentaron las bases, para posteriormente, confirmar que la técnica era posible, viable y segura. Fundó la Asociación Americana de Nutrición Parenteral y Enteral (ASPEN, por su sigla en Inglés), de la que fue el primer presidente y primer miembro honorario. Dedicó sus esfuerzos a la nutrición, cirugía, enseñanza e investigación, a sus alumnos, residentes, colaboradores, amigos y familia hasta sus últimos días. Excelente ser humano, será recordado por sus cualidades, su genial aporte a la medicina que ha hecho que un sinnúmero de pacientes sobrevivan y mejoren la calidad de vida.

En este artículo se presenta una semblanza de su vida, sus logros personales
\end{abstract}

\section{Summary}

Professor Stanley J Dudrick, is considered the father of Parenteral Nutrition, or nutrition by vein. He was a pioneer in the nutrient administration totally by vein. His first works performed in dog puppies set the bases to confirm that this technique was feasible, possible and safe. He founded the first multidisciplinary society, The American Society for Parenteral and Enteral Nutrition (ASPEN) of which he was elected the first and honorary president. He dedicated his entire effort to nutrition, surgery, and to his patients, students, residents, colleagues, friend and family until his last days. Excellent human being, he will be remembered for his qualities, his brilliant contribution to medicine that has made countless patients survive and improve the quality of life.

This article is presented as a semblance of his life, his success story both personally and academically and it is based on a

\section{Resumo}

O professor Stanley J. Dudrick, considerado o pai da nutrição parenteral ou intravenosa, foi pioneiro no uso desta via para a administração de nutrientes. Seus primeiros estudos em cães lançaram as bases, para confirmar posteriormente que a técnica era possível, viável e segura. Fundou a Associação Americana de Nutrição Parenteral e Enteral, ASPEN (sigla em inglês), de quem foi o primeiro presidente e membro honorário. Dedicou seus esforços, à nutrição, cirurgia, ensino e pesquisa, aos seus alunos, residentes, colaboradores, amigos e familiares até aos seus últimos dias. Grande ser humano, ele será lembrado por suas qualidades, sua grande contribuição à medicina, que fez inúmeros pacientes sobreviverem e meIhorarem a qualidade de vida.

Este artigo apresenta um relato da sua vida, suas realizações pessoais e acadêmicas, 
y académicos basada en conversaciones personales realizadas a partir de 1995 hasta el mes de noviembre de 2019 cuando visité su casa en el estado de New Hampshire, EUA.

Palabras clave: nutrición parenteral, falla intestinal, historia, semblanza. couple of interviews and experiences that he shared with me since 1995 up to two months before the end of his life in his home up in New Hampshire, USA.

Keywords: Parenteral nutrition; Intestinal failure history; Semblance. baseadas em conversas pessoais, feitas entre 1995 e dois meses atrás (novembro 2019), quando visitei sua casa no estado de New Hampshire, EUA.

Palavras-chave: nutrição parenteral, insuficiência intestinal, história, relato.
Jefe del servicio de Nutrición Clínica Hospital General de México, Ciudad de México.

\section{INFANCIA Y ESTUDIOS DE MEDICINA}

El Profesor Stanley J Dudrick, considerado el padre de la nutrición parenteral o endovenosa nació en Nanticoke, Pensilvania, el 9 de abril de 1935. Su pueblo era considerado uno de los más importantes por la explotación de minas de carbón, muchos de sus familiares cercanos trabajaban en esta industria, incluyendo su padre y su abuelo. Él contaba que su padre le enseñó, siendo chico, a trabajar en varios oficios, desde la construcción, la mecánica automotriz, hasta algo de minería (Figura 1).

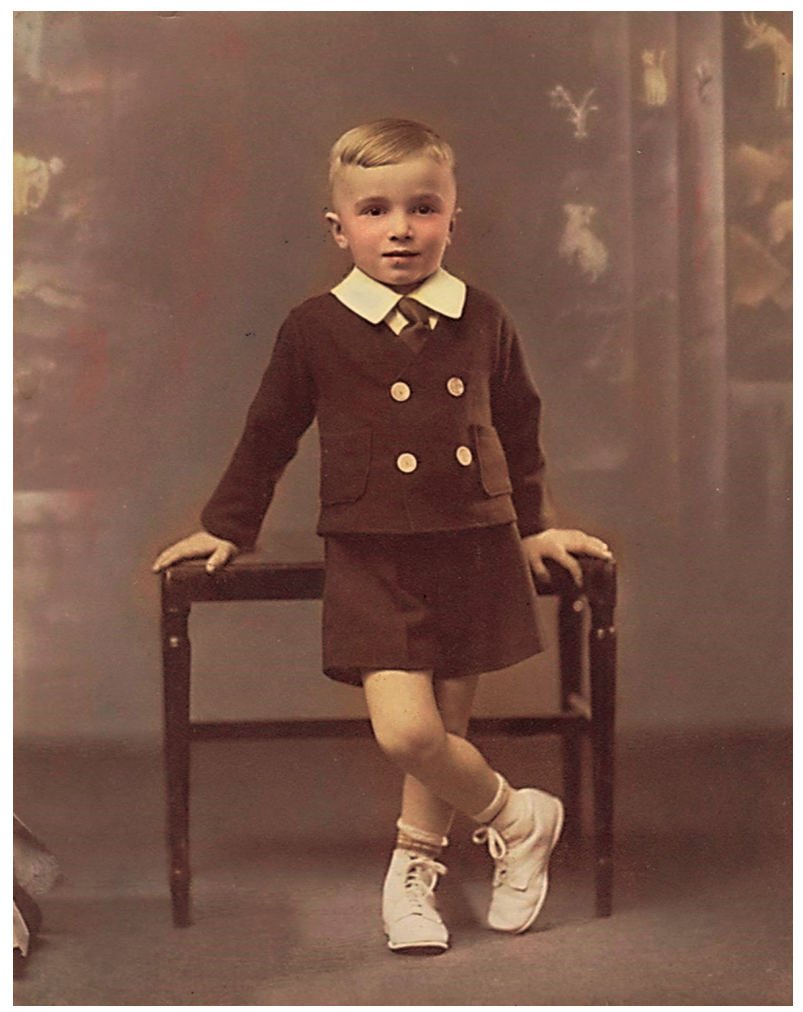

Figura 1. Stanley J. Dudrick de 3 años 9 meses, en 1939.
*Correspondencia: Vanessa Fuchs-Tarlovsky vanessafuchs@hotmail.com

Su madre se dedicaba al hogar, y dado que su pueblo era muy pequeño tuvo el privilegio de crecer rodeado de ambas familias, siendo el primer niño de la familia tuvo el cariño y apoyo de todos. Cuando tenía apenas 6 años y su hermana 2, su madre enfermó de fiebre reumática. Entonces no había penicilina por lo que la mayoría de los pacientes con esta enfermedad morían. Recuerda que en esa época varios médicos generales rurales iban a visitar con frecuencia a su madre enferma. Cada vez que ellos iban, su familia lo hacía vestirse formal para recibirlos, por lo que él se formó la idea de que debían ser gente importante y los admiraba mucho, admiración que aumentó cuando milagrosamente su madre sobrevivió a la enfermedad a pesar de que la familia estaba preparada para lo peor. Es en ese momento cuando decidió que a eso dedicaría su vida, que estudiaría medicina y así lo hizo.

Realizó sus estudios básicos en su ciudad natal y los estudios universitarios en el Frankin and Marshall College, en Lancaster, Pensilvania (1953 - 1957). Refiere que Lancaster era un pueblo extremadamente limpio, lleno de granjas de migrantes holandeses y alemanes muy ordenados, y que en verdad disfrutó esa etapa de su vida. Fue ahí donde entró por primera vez a un laboratorio de investigación, en el cual solían hacer crecer tomates con hidroponía, lo que le recordaba su infancia en su granja ya que además de mineros, su familia también sembraba alimentos que posteriormente usaban como fuente de sustento.

Él ya sabía que al terminar la universidad iba a ingresar a una escuela de medicina, la entrevista con el director de la escuela de medicina de la universidad de Pensilvania en Filadelfia, donde quería ingresar, fue excelente y con eso aseguró que no solo quería estudiar medicina sino trabajar en esa escuela cuando terminara sus estudios. Cuando llegó el momento de aplicar, únicamente envió 
solicitud a dos escuelas, la Universidad de Pensilvania (PENN) y al Jefferson College of Medicine. En las dos instituciones fue aceptado; sin embargo, optó por la primera, iniciando su vida académica en PENN. De la facultad de medicina de esta universidad (1957 - 1961) obtuvo su título de médico (MD).

Al graduarse como médico, decidió continuar con la residencia en cirugía e inició su internado en el hospital de la Universidad de Pensilvania en 1961, en donde se enfocó en cirugía general y trabajó como asistente de un profesor desde 1962 hasta 1966. En ese lugar, dos personajes tuvieron gran influencia en la carrera del doctor Dudrick, los doctores Jonathan E. Rhoads y I.S. Ravdin (Figura 2).
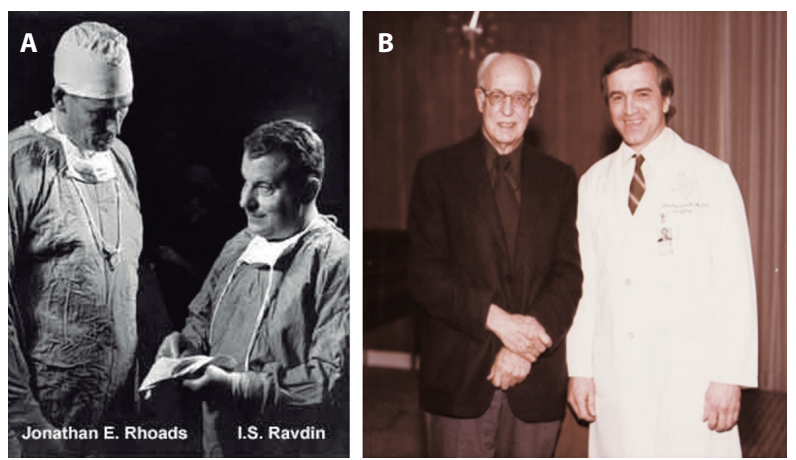

Figura 2. A. Doctores Jonathan E. Rhoads y I.S. Ravdin. B. Harry M. Vars, PhD, with SJD mientras se desempeñaba como profesor visitante de bioquímica en cirugía en la Facultad de medicina de la Universidad de Texas en Houston a mediados de la década de 1970

\section{RESIDENCIA DE CIRUGÍA Y EL AMOR POR LA NUTRICIÓN}

Desde entonces mostró gran sensibilidad y preocupación por los enfermos que, a pesar de que el procedimiento quirúrgico fuera realizado de manera impecable, se complicaban porque no había manera de alimentarlos y aportar lo necesario para conservar o mejorar su estado nutricional, cicatrización, infecciones, días de estancia hospitalaria y morbi-mortalidad. De manera sistémica, estos pacientes llegaban en malas condiciones generales y nutricionales, y aunado a eso, requerían una nueva intervención quirúrgica. Sin embargo, debido a las condiciones en que llegaban, muchos de ellos morían por la propia enfermedad y por imposibilidad de resistir una nueva cirugía y el trauma que esta representaba.

En aquel entonces no había nada que hacer en esos casos, excepto hablar con los familiares acerca de las condiciones del paciente y tomar decisiones difíciles.
Las heridas no cicatrizaban, los pacientes carecían de fuerzas y se presentaban varias complicaciones con desenlaces funestos e indeseables.

Incluso, Stanley Dudrick refirió, que un día en el cual murieron tres pacientes de su profesor Rhoads, quienes habían sido intervenidos quirúrgicamente con una excelente técnica y; sin embargo, no hubo forma de salvar su vida, pensó en no dedicarse más a esto. Sostuvo esta conversación con el mismo Rhoads concluyendo que la muerte de estos pacientes se debía a su condición nutricional y que no había forma de alimentarlos ya que el intestino no funcionaba. Dudrick insistía en que debía haber alguna forma y trató, en varios adultos, de introducir soluciones cada vez más concentradas, hasta la flebitis. Usaron los lípidos disponibles en aquella época, los cuales contenían partículas grandes que ocasionaban oclusión de las venas utilizadas. Además, tampoco existían los diuréticos lo suficientemente eficaces para liberar el exceso de volumen de líquidos.

Ante estas circunstancias, el joven residente de 33 años, durante su año dedicado a investigación como parte de su programa de residencia quirúrgica, decidió llevar a cabo un ensayo el cual consistía en estudiar la forma de llevar a cabo el trabajo del tubo digestivo de manera "artificial" o externa, esto es, digerir los alimentos hasta la mínima expresión e introducirlos directamente a la sangre, haciéndolos accesibles para ser aprovechados por las células del organismo y realizar el proceso de nutrición. El único antecedente era la utilización de soluciones glucosadas, las cuales por su dilución y bajo aporte nutricional, eran poco efectivas.

Stanley expuso su idea a su maestro, el doctor Jonathan Rhoads, quien en un inicio consideró que la idea era muy interesante, pero muy difícil de lograr; en primer lugar, porque los nutrientes tenían muy alta osmolaridad y ninguna vena lo toleraría y, en segundo lugar, por el enorme riesgo de infección que significaba tener acceso directo a una vena, lo cual podría tener consecuencias fatales.

A pesar de esos comentarios, Dudrick comenzó a infundir algunos nutrientes directamente disueltos en una vena del brazo, diluidos en una gran cantidad de agua. La cantidad energética que pudo pasar fue muy pequeña y la cantidad de agua excesiva. Logró concentrar la cantidad aportada un poco más pero el resultado fue fallido por la osmolaridad de la mezcla que la vena periférica no toleró.

Posteriormente, pensó en utilizar venas de mayor calibre, lo que resultaría peligroso, pues la única técnica conocida entonces para la instalación de un catéter 
con el fin de infundir sustancias era la venodisección. El doctor Rhoads le propuso hacerlo en la vena inguinal, pero le recordó que un catéter puesto en esa zona tendría enormes posibilidades de infección. Dudrick pensó entonces en la subclavia o la yugular; ambas tenían ventajas y desventajas. La yugular es una vena de acceso más fácil, pero debido a su ubicación en el cuello resulta un sitio muy incómodo para la colocación del catéter, siendo una zona más delicada y de mucho movimiento. Sin embargo, la subclavia era casi imposible de alcanzar debido al lugar anatómico en donde se encuentra, ya que es muy difícil practicar una venodisección del misma. Por estas dificultades decidió iniciar sus experimentos en perros a los que introdujo, por venodisección, un catéter en la yugular.

Para esta época el doctor Dudrick ya era jefe de residentes e instructor de cirugía en el Hospital de la Universidad de Pensilvania. Procedió a planear el experimento, a organizar la formulación y la estabilidad de los nutrientes que infundiría, para lo cual contó con el apoyo del farmacéutico de la Universidad, doctor Harry Vars (Figura 3).

Se planeó el experimento y se consiguieron ocho cachorros de raza Beagle; a cuatro de ellos se les alimentó por vía oral mediante dieta a base de una especie de croqueta, controlando su contenido y aporte nutricional, y a los otros cuatro se les instaló un catéter para infundirles la solución a base de la mezcla parenteral. La Figura 4 muestra los bosquejos originales que el doctor Dudrick diseñó y presentó para el desarrollo de su proyecto.

Esta raza de perros fue elegida en primer lugar por tener pelo corto, lo cual facilitaba el manejo del catéter y en segundo lugar por su carácter tranquilo y además por tener una anatomía y metabolismo más parecida al humano según comentó Dudrick en numerosas ocasiones. Cabe mencionar que se requirieron fondos para la investigación ya que cada cachorro costó alrededor de 300 dólares de esa época. Hacía énfasis también, en que en la actualidad es muy difícil imaginar cómo eran los primeros catéteres: grandes, incómodos, peligrosos y de un solo lumen.

Es evidente que se intentó hacer crecer a los cachorros con alimentación endovenosa más de una vez, de hecho, los primeros que intentó alimentar fueron separados de la madre muy prematuramente (al nacimiento), desarrollaron micro cálculos uretrales que le bloquearon la uretra y causaron su muerte (se cree que no haber permitido a la madre limpiarlos, lo que naturalmente hace, ocasionó el fracaso). Poco a poco fue perfeccionando la técnica, adaptando los catéteres con los materiales que tenía o que lograba conseguir en tlapalerías (ferreterías) y esterilizarlos; finalmente tuvo que lidiar con bacterias y hongos para lo cual también ideó la forma de adecuar y proporcionar ungüentos que contenían antimicóticos y antibióticos hasta que logró controlar el proceso.

$\mathrm{Al}$ cabo de 257 días, del estudio final con los ocho perritos, pudo demostrar que el crecimiento de cacho-
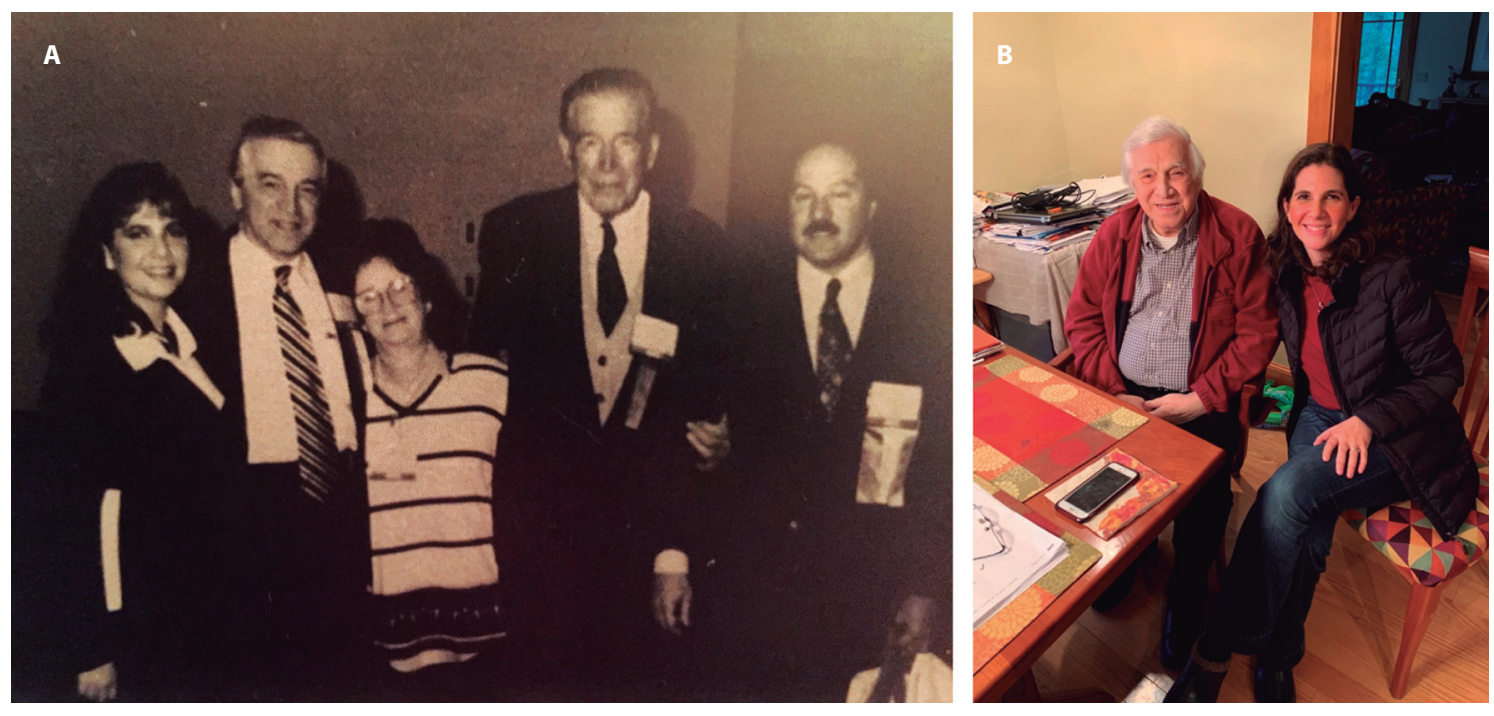

Figura 3. A. Doctor SJ Dudrick y Vanessa Fuchs en el congreso ASPEN 1995. B. Visita a casa del Doctor SJ Dudrick en New Hampshire, USA, en noviembre 2019. 
rros alimentados por vía endovenosa era equivalente al proceso de alimentación oral, lo sorprendente fue que crecían al mismo ritmo y sin ningún problema visible, lo que permitió seguir adelante con el proyecto. Fue con este estudio y sus resultados que demostró que la alimentación parenteral era posible y factible. Publicó este artículo en 1967 en la revista Surgical Forum ${ }^{(1)}$.

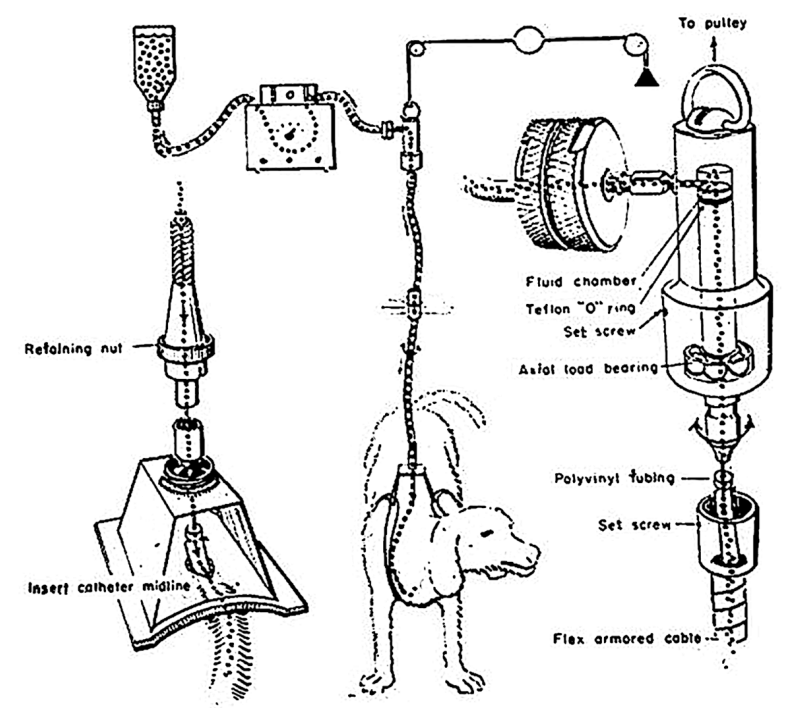

Figura 4. Diagrama de representación del aparato usado para infundir a los cachorros Beagle a través de la vena cava superior la nutrición parenteral de manera continua.

En el año 1966, los doctores Dudrick y Rhoads asistieron al Congreso Mundial de Nutrición en Hamburgo, Alemania, donde presentaron por primera vez ante el mundo el hallazgo que se tituló "Growth of puppies receiving all nutritional requirements by vein"

Debido al interés que causó este estudio, a su regreso decidió diseñar otro experimento, esta vez con doce cachorros, y a partir de entonces se enfocó en la posibilidad de probar esta técnica en seres humanos.

El doctor Dudrick siempre hizo énfasis en que a diferencia de lo que está escrito en los libros, revistas y de lo que piensa todo el mundo, antes de probar este método en la niña recién nacida que todos conocen, comenzó a probar esta técnica en adultos.

En un principio instaló el catéter en la vena yugular, lo cual fue difícil con los conocimientos y el tipo de catéteres disponibles en esa época, aún más complicado en pacientes de sexo masculino, debido al crecimiento de la barba. Después de un tiempo, aplicó la técnica que se conoce como punción percutánea, la cual había leído en un antiguo artículo publicado en " $\mathrm{La}$
Presse Medicale" (1952) por un médico militar llamado Aubanic durante la Segunda Guerra Mundial ${ }^{(1)}$. El doctor Rhoads estaba escéptico debido a que esta técnica podía ocasionar perforación de la pleura, pero Dudrick insistía en que, si se llevaba a cabo de manera adecuada y con cuidado, tenía que resultar. Realizó por primera vez la punción con éxito en un paciente que se alimentó de esta forma por un tiempo. Con el fin de evitar infecciones del catéter se hicieron necesarias investigaciones profundas sobre antibióticos, no disponibles en aquella época. Una vez que se logró combatir este problema bacteriano, surgieron los hongos. "En fin" comenta, "no fue fácil, pero el beneficio que se consiguió estuvo muy por encima de todos estos detalles".

En 1968 escribió el artículo, un clásico sobre este tema, en el cual incluyó el estudio de los 12 cachorros y el de seis personas adultas a quienes se alimentó con éxito de esta forma. En este estudio, se estableció una serie de normas, principalmente de higiene en el manejo de los catéteres centrales. Dudrick presentó por primera vez esta investigación en un congreso llevado a cabo en Nueva York y la publicó con el título de "Long term parenteral nutrition with growth, developement and positive nitrogen balance" en la revista Surgery ${ }^{(2)}$.

Ese año Stanley J. Dudrick ya era profesor asociado e investigador en el Departamento de Cirugía experimental, y hacía parte del equipo científico del mismo.

A raíz de estos trabajos, llegaron médicos y científicos de distintas partes del mundo para aprender la colocación de los catéteres y la alimentación para los pacientes que lo requerían. Sin embargo, se publicaron a su vez numerosos artículos que concluían que esta nueva técnica era un peligro. El doctor Dudrick estaba convencido de que el problema que registraban estos artículos derivaba de una mala aplicación de la técnica o bien de no seguir las indicaciones precisas establecidas desde el inicio, ya que las infecciones y diversas complicaciones fueron frecuentes. Refiere con pesar que para él, "esa fue una época muy difícil y decepcionante", y agrega "en ese entonces llegué a pensar que todo lo que habíamos hecho no servía para nada”.

\section{EL CASO DE LA PRIMERA RECIÉN NACIDA ALIMENTADA EXCLUSIVAMENTE POR VENA}

Fue entonces cuando se presentó una circunstancia fortuita. En el Children's Hospital de la Universidad de Pensilvania nació una niña con un problema genético intestinal parecido a la atresia intestinal, es decir con incapacidad permanente para usar el tracto digestivo 
para alimentarse, lo afortunado del suceso fue que la niña nació en dicho hospital, de lo contario hubiera muerto a los pocos días de nacida sin poder hacer nada al respecto. Un estudiante que había sido interno en el Hospital de la Universidad de Pensilvania con el doctor Dudrick escuchó acerca de su experimento con los perros y lo contactó para solicitarle una sesión general en su hospital, ya que se encontraba de residente de cirugía pediátrica y esa bebé era su paciente. El aceptó encantado y posterior a la conferencia le comentaron el caso y preguntaron si estaba dispuesto a usar esta forma experimental de alimentación en esta bebé. Al principio lo dudó ya que el hospital estaba lejos del laboratorio y del hospital de adultos y no le daría tiempo. Fue entonces cuando involucró al doctor Douglas Willmore, quien vivía cerca del hospital y se responsabilizó del cuidado y supervisión del caso.

Después de trámites dispendiosos, ya que no existían los comités de ética como hoy en día, se permitió al doctor Dudrick instalar un catéter de la misma forma y por una vena central como se aprecia en la Figura 5, y alimentar a la niña por vía endovenosa. Gracias al cuidado del catéter, al uso de medicamentos antimicrobianos y antifúngicos, y a la atención que todo el equipo ofreció a la niña, esta bebé logró crecer de manera normal ${ }^{(3)}$.

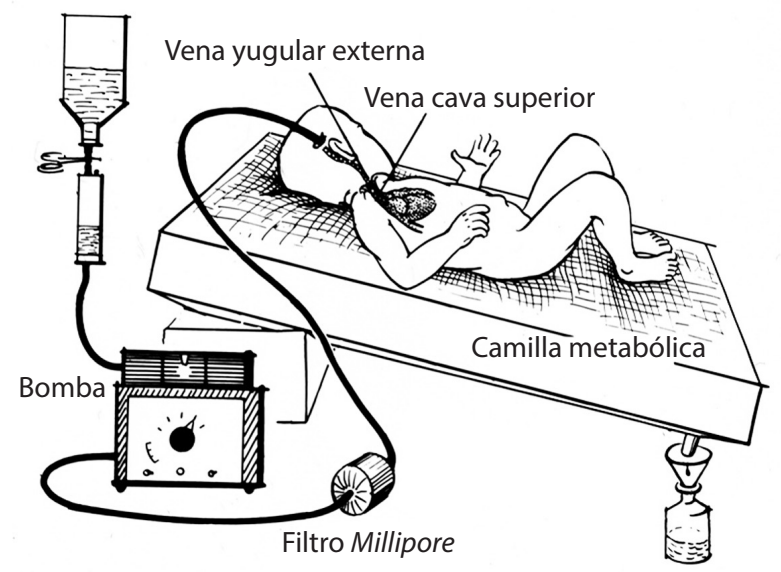

Figura 5. Se muestran los componentes de la técnica de alimentación intravenosa en lactantes y el lecho metabólico especial diseñado para la recolección de orina y heces sin catéteres invasivos o dispositivos de recolección engorrosos.

Sin embargo, después de algún tiempo, los directivos del hospital decidieron dejar de alimentarla, ya que debía irse a casa y aun no existía la tecnología para el home care con este tratamiento. El hecho de suspenderle la alimentación causó la desnutrición que le produjo la muerte. Pese a que el doctor Dudrick no estuvo de acuerdo con la difícil decisión tomada por los médicos tratantes y la parte administrativa, aceptó que la bebé no era su paciente y, al faltar la parte ética, y a que ciertos procedimientos no estaban aún regulados como hoy en día, lamentablemente no pudo hacer nada más.

También comentó que gracias a esta niña aprendieron mucho de la forma de alimentar pacientes por vía endovenosa, desde la deficiencia de lípidos, que no se contaba con tecnología probada para administrarlos, de vitamina $\mathrm{D}$, ya que pese al calcio que se le proporcionaba, tuvo una fractura que demostró que se requerían vitaminas, minerales endovenosos y zinc, entre otros.

Dijo Dudrick: "Esa fue la clave para demostrarle al mundo entero que la técnica efectivamente servía, y que todos aquellos fracasos que publicaban se debían al mal seguimiento de las instrucciones planteadas". El artículo sobre el caso de la niña se publicó en el Journal of the American Medical Association (JAMA) titulado "Growth of an infant receiving all nutrients by vein"(4). También comentó que de esa paciente se aprendió mucho sobre nutrición y sobre el metabolismo, aún hay una placa dedicada a ella y a su familia en el hospital de niños de la Universidad de Pensilvania.

\section{LA FUNDACIÓN DE ASPEN}

De 1968 - 1972 Stanley Dudrick fue el jefe del departamento de cirugía del Philadelphia Veterans Administration Hospital. En este último año, fundó, y fue su primer presidente, la Asociación Americana de Alimentación Parenteral y Enteral (ASPEN), sigue vigente y creciendo en forma importante, celebrando un congreso anual y publicando revistas de alta calidad. Actualmente, cuenta con revistas de alto nivel académico como el Journal of Parenteral and enteral nutrition $y$ en Nutrition in Clinical Practice. El ASPEN's Stanley J. Dudrick Research Award que se entrega cada año durante el Dudrick's symposium se creó para estimular la investigación en el área de la nutrición ${ }^{(5)}$.

A partir de 1972 y hasta 1980 fue jefe del departamento de cirugía de la Escuela de Medicina de la Universidad de Texas, Houston y jefe de servicios quirúrgicos en el Hermann Hospital. Fungió como profesor de cirugía en el St. Luke's Episcopal Hospital y consultor de cirugía del Texas Cancer Center y el MD Anderson Hospital and Tumor Institute (1972 - 1978). En 1981 ingresó a la planta del Texas Children's Hospital.

En 1988 regresó a su estado natal donde llegó a ser director general del Pennsylvania Hospital. De 1990 1993 regresó al Texas Cancer Center para convertirse 
en el primer jefe del servicio de soporte nutricional del Hermann Hospital y director del centro científico de Nutrición, así como del centro de enfermedades cardiovasculares del mismo hospital. Posteriormente hasta 1993, fue profesor de cirugía en la Escuela de Medicina de la Universidad de Pennsylvania.

\section{LOS AVANCES}

Poco a poco, el sistema de alimentación endovenosa o nutrición parenteral fue mejorando y modernizándose. El doctor Dudrick comentó que las primeras bombas de infusión se diseñaron en su oficina y que de ninguna manera se parecían a las que tenemos hoy ${ }^{(6)}$.

En la actualidad el progreso tecnológico y el conocimiento, no solo de los materiales utilizados en catéteres y dispositivos, sino también de infecciones intrahospitalarias, sistema venoso, es evidente. Otro gran avance tecnológico se ha dado en las soluciones comerciales con una gran variedad de contenidos, fuentes, marcas $\mathrm{y}$ concentraciones.

Este descubrimiento dio lugar al desarrollo de diversas industrias y por supuesto, a la explotación comercial de las mismas. Sin embargo, su descubridor no recibió importantes sumas de dinero por este invento, pues, tal y como el mismo Dudrick dijo: "Si hubiera aceptado una remuneración importante, todos mis esfuerzos hubieran perdido credibilidad científica, y mi objetivo era demostrar que la alimentación parenteral era posible para mejorar la calidad de vida y el tiempo de vida de los pacientes que requerían este tipo de apoyo"

Y agregó: "Lo más importante, ante todo, es que muchos pacientes viven $y$ tienen mayores posibilidades de recuperación de sus padecimientos gracias a este sistema, y de no existir, muchos no estarían aquí para contarlo".

Algunos de sus últimos trabajos los desempeñó en el estado de Connecticut: en el Bridgeport Hospital, fue director del Programa de Cirugía del St. Mary's
Hospital en Waterbury, afiliado al programa de Cirugía de la Universidad de Yale, de la cual fue miembro de la Academia.

El doctor Dudrick publicó más de 100 artículos en revistas científicas; recibió más de 60 membrecías honoríficas y reconocimientos en diferentes partes del mundo.

Resulta interesante constatar la manera en que la vida de una persona puede aportar tanto a la humanidad. El legado del doctor Stanley J. Dudrick y de las personas que siguieron sus pasos ha sido clave para aumentar las posibilidades de recuperación de diversos padecimientos y sus respectivas complicaciones, como son las fístulas enterocutáneas, pancreatitis grave y síndromes de intestino corto y falla intestinal, entre otras. Además, de contribuir a mejorar la calidad de vida de un gran número de pacientes en el mundo.

\section{Referencias bibliográficas}

1. Aubaniac R. L'injection intraveneuse sous claviculaire. La Presse Médicale. 1952; 60:1456.

2. Dudrick SJ, Wilmore DW, Vars HM. Long-term total parenteral nutrition with growth in puppies and positive nitrogen balance in patients. S. Forum 18: 356, 1967.

3. Dudrick SJ, Willmore DW, Vars HM, Rhoads JE. Long term total parenteral nutrition with growth, development, and positive nitrogen balance. Surgery. 1968 (64):131-41.

4. Wilmore DW, Dudrick SJ. Growth and development of an infant receiving all nutrients exclusively by vein. JAMA. 1968;203:860-4.

5. Fuchs-Tarlovsky V. Historia de la Nutrición Parenteral y Stanley Jonathan Dudrick. Cuadernos de Nutrición. 1996;15(5):36-9.

6. Gosche JR. Pediatric History Center. Oral History Project. Stanley J. Dudrick. 2007. Naugatuck, Connecticut. American Academy of Pediatrics. Elk Grove Village, IL. [Internet] (Consultado el 26 de enero 2020). Disponible en https:// www.aap.org/en-us/about-the-aap/Pediatric-HistoryCenter/Documents/Dudrick.pdf 